\title{
Fractional Fourier transform in information optics
}

\section{David Mendlovic, Zeev Zalevsky}

David Mendlovic, Zeev Zalevsky, "Fractional Fourier transform in information optics," Proc. SPIE 3831, Sixth International Conference on Education and Training in Optics and Photonics, (16 June 2000); doi: 10.1117/12.388699 


\title{
The Fractional Fourier Transform in Information Optics
}

\author{
David Mendlovic and Zeev Zalevsky
}

Tel-Aviv University, Faculty of Engineering, 69978 Tel-Aviv, Israel

\begin{abstract}
The fractional Fourier transform is an important tool for both signal processing and optical communities. This paper presents a tutorial which includes the major related aspects of this transformation.
\end{abstract}

\section{Introduction}

The fractional Fourier transform (FRT) operation was shown to be useful for various spatial filtering and signal processing applications [1-8]. The FRT is a private case of the $A B C D$ matrix. When the $A B C D$ matrix accepts the form of:

$$
\left[\begin{array}{ll}
\mathrm{A} & \mathrm{B} \\
\mathrm{C} & \mathrm{D}
\end{array}\right]=\left[\begin{array}{cc}
\cos \phi & -\sin \phi \\
\sin \phi & \cos \phi
\end{array}\right]
$$

the ABCD transform becomes the FRT.

In this transform the amount of shift variance may be controlled by choosing the proper fractional order $p$ for the transformation while $\phi$ equals to $p \pi / 2$. When the fractional order is one, the FRT becomes the conventional Fourier transform that is totally shift invariant. For fractional order of zero the FRT gives the input function, i.e. totally shift variant. For any other fractional orders in between, the transform has a partial amount of shift variance. 


\section{FRT- Definition}

There are two common interpretations for the FRT. Both definitions were proven to be identical as shown in Ref. [7].

\subsection{Definition based on propagation in graded index media}

The first FRT definition [9-11] is based on the field propagating along a quadratic graded index (GRIN) medium having a length proportional to $p$ ( $p$ being the FRT order). The eigen-modes of quadratic GRIN media are the Hermite-Gaussian (HG) functions, which form an orthogonal and complete basis set. The $\mathrm{m}$ th member of this set is expressed as:

$$
\Psi_{\mathrm{m}}(\mathrm{x})=\mathrm{H}_{\mathrm{m}}\left(\frac{\sqrt{2} \mathrm{x}}{\omega}\right) \exp \left(-\frac{\mathrm{x}^{2}}{\omega^{2}}\right)
$$

where $\mathrm{H}_{\mathrm{m}}$ is a Hermite polynomial of order $\mathrm{m}$ and $\omega$ is a constant associated with the GRIN medium parameters. An extension to two lateral coordinates $x$ and $y$ is straightforward, with $\psi_{\mathrm{m}}(\mathrm{x}) \psi_{\mathrm{n}}(\mathrm{y})$ as elementary functions.

The propagation constant for each HG mode is given by:

$$
\beta_{\mathrm{m}}=\mathrm{k} \sqrt{1-\frac{2}{\mathrm{k}} \sqrt{\frac{\mathrm{n}_{2}}{\mathrm{n}_{1}}}\left(\mathrm{~m}+\frac{1}{2}\right)} \approx \mathrm{k}-\sqrt{\frac{\mathrm{n}_{2}}{\mathrm{n}_{1}}}\left(\mathrm{~m}+\frac{1}{2}\right)
$$

with $k=2 \pi / \lambda$ and $\lambda$ is the wavelength. The HG set is used to decompose any arbitrary distribution $\mathrm{u}(\mathrm{x})$

$$
\mathrm{u}(\mathrm{x})=\sum_{\mathrm{m}} \mathrm{A}_{\mathrm{m}} \Psi_{\mathrm{m}}(\mathrm{x})
$$

where the coefficient $A_{m}$ of each mode $\psi_{m}(x)$ is given by:

$$
A_{m}=\int_{-\infty}^{\infty} u(x) \Psi_{m}(x) / h_{m} d x
$$

with

$$
\mathrm{h}_{\mathrm{m}}=2^{\mathrm{m}} \mathrm{m} ! \sqrt{\pi} \omega / \sqrt{2}
$$

Using the above decomposition, the FRT of order $p$ is defined as:

$$
\begin{gathered}
\Im^{\mathrm{p}}[\mathrm{u}](\mathrm{x})=\sum_{\mathrm{m}} \mathrm{A}_{\mathrm{m}} \Psi_{\mathrm{m}}(\mathrm{x}) \exp \left(\mathrm{i} \beta_{\mathrm{m}} \mathrm{pL}\right) \\
\mathrm{L}=\frac{\pi}{2} \sqrt{\frac{\mathrm{n}_{1}}{\mathrm{n}_{2}}}
\end{gathered}
$$

$\mathrm{L}$ is the GRIN length that results in the conventional Fourier transform. It was shown [10] that this definition agrees well with the classical Fourier transform definition when $\mathrm{p}=1$. 


\subsection{Definition based on Wigner distribution function}

A complete signal description, displaying space and frequency information simultaneously, can be achieved by the space-frequency Wigner distribution function (WDF) [12].

In Ref. [13] the fractional Fourier transform operation is defined by following the signal $\mathrm{u}(\mathrm{x})$ while its WDF is rotated by an angle $\phi=\mathrm{p} \pi / 2$. Note that the WDF of a $1-\mathrm{D}$ function is a 2-D function and the rotation interpretation is easily displayed.

In Ref. [13], the same rotation strategy was generalized to 2-D signals, i.e. images, whose WDFs are 4-D distributions. The WDF of a function can be rotated with bulk optics [13].

This optical setup represents in the WDF space three shearing operations: $\mathrm{x}, v$-shearing or $v, \mathrm{x}$-shearing. Where $v$ is the spectral coordinate and $\mathrm{x}$ is the spatial one. The $\mathrm{x}$-shearing is performed by free-space propagation, then a lens performs $v$-shearing, then again $x$-shearing is performed by free-space propagation. By analyzing the optical configuration of Ref. [13], Lohmann obtained:

$$
u_{p}(x)=\mathfrak{J}^{p}\left[u\left(x_{0}\right)\right]=C_{1} \int u\left(x_{0}\right) \exp \left(i \pi \frac{x_{0}^{2}+x^{2}}{\lambda f_{1} \tan \phi}\right) \exp \left(-i 2 \pi \frac{x_{0}}{\lambda f_{1} \sin \phi}\right) d x_{0}
$$

with

$$
\mathrm{C}_{1}=\frac{\exp \left[-\mathrm{i}\left(\frac{\pi \operatorname{sgn}(\sin \phi)}{4}\right)-\frac{\phi}{2}\right]}{\sqrt{\left|\lambda \mathrm{f}_{1} \sin \phi\right|}}
$$

This last equation defines the FRT for 1-D functions. Generalization for 2-D functions is straightforward. Note that $\lambda f_{1}$ is also coined the scaling factor.

The two interpretations of the FRT operation have been united into one formulation through a transformation kernel, as illustrated in Ref. [8]:

$$
u_{p}(x)=\mathfrak{J}^{p}\left[u\left(x^{\prime}\right)\right](x)=\int B_{p}\left(x, x^{\prime}\right) u\left(x^{\prime}\right) d x^{\prime}
$$

where $\mathrm{B}_{\mathrm{p}}\left(\mathrm{x}, \mathrm{x}^{\prime}\right)$ is the kernel of the transformation and $\mathrm{p}$ is the fractional order. The kernel has two optical interpretations, one as a propagation through GRIN medium [10]

$$
\mathrm{B}_{\mathrm{p}}\left(\mathrm{x}, \mathrm{x}^{\prime}\right)=\sqrt{2} \exp \left[-\frac{\mathrm{x}^{2}+\mathrm{x}^{\prime 2}}{\omega}\right] \sum_{n=0}^{\infty} \frac{\mathrm{i}^{-\mathrm{pn}}}{2^{\mathrm{n}} \mathrm{n} !} \mathrm{H}_{\mathrm{n}}\left(\frac{\sqrt{2} \mathrm{x}}{\omega}\right) \mathrm{H}_{\mathrm{n}}\left(\frac{\sqrt{2} \mathrm{x}^{\prime}}{\omega}\right)
$$

and the second as a rotation operation applied over the Wigner plane [13]

$$
B_{p}\left(x, x^{\prime}\right)=C_{1} \exp \left[i \pi\left(\frac{x^{2}+x^{\prime 2}}{\lambda f_{1} \tan \phi}\right)-2 \pi i\left(\frac{x^{\prime}}{\lambda f_{1} \sin \phi}\right)\right]
$$


Note that $\omega$ is the coefficient that connects the two interpretations:

$$
\omega=\sqrt{\frac{\lambda f_{1}}{\pi}}
$$

\section{Properties of the FRT}

- Linearity:

The FRT of a linear combination of two input functions $u_{1}$ and $u_{2}$ behaves according to the definition of linear systems. $c_{1}$ and $c_{2}$ are constants.

$$
\mathfrak{J}^{\mathrm{p}}\left[\mathrm{c}_{1} \mathrm{u}_{1}(\mathrm{x})+\mathrm{c}_{2} \mathrm{u}_{2}(\mathrm{x})\right]=\mathrm{c}_{1} \mathfrak{J}^{\mathrm{p}}\left[\mathrm{u}_{1}(\mathrm{x})\right]+\mathrm{c}_{2} \mathfrak{J}^{\mathrm{p}}\left[\mathrm{u}_{2}(\mathrm{x})\right]
$$

- Continuity:

Two FRTs with different orders $p_{1}$ and $p_{2}$ yield the following theorem:

$$
\mathfrak{J}^{\left(c_{1} p_{1}+c_{2} p_{2}\right)}[u(x)]=\mathfrak{J}^{c_{1} p_{1}}\left[\mathfrak{J}^{c_{2} p_{2}}(u(x))\right]=\mathfrak{J}^{c_{2} p_{2}}\left[\mathfrak{I}^{c_{1} p_{1}}(u(x))\right]
$$

- Parseval's theorem:

$$
\int\left|u_{0}\left(x_{0}\right)\right|^{2} d x_{0}=\int\left|u_{p}\left(x_{p}\right)\right|^{2} d x_{p}
$$

- Shift theorem:

If the input object is shifted by the amount of a, then its FRT yields:

$\mathrm{u}_{\mathrm{p}}(\mathrm{x} ; \mathrm{a})=\mathfrak{J}^{\mathrm{p}}\left[\mathrm{u}_{0}\left(\mathrm{x}_{0}+\mathrm{a}\right)\right]=\exp [\mathrm{i} \pi \mathrm{a} \sin \phi(2 \mathrm{x}+\mathrm{a} \cos \phi)] \mathrm{u}_{\mathrm{p}}(\mathrm{x}+\mathrm{a} \cos \phi)$

- Scaling theorem:

If the input object is scaled by the factor of a, then its FRT yields:

$$
\mathrm{u}_{\mathrm{p}}(\mathrm{x} ; \mathrm{a})=\mathfrak{J}^{\mathrm{p}}\left[\mathrm{u}_{0}\left(\mathrm{ax}_{0}\right)\right]=\frac{\Psi}{\mathrm{a}} \mathrm{u}_{\hat{\mathrm{p}}}\left(\frac{\mathrm{x}}{\sqrt{\sin ^{2} \phi\left(\mathrm{a}^{2}-\frac{1}{\mathrm{a}^{2}}\right)+\frac{1}{\mathrm{a}^{2}}}}\right)
$$

where

$$
\begin{array}{r}
\hat{\mathrm{p}}=\frac{2}{\pi} \tan ^{-1}\left[\mathrm{a}^{2} \tan \left(\frac{\pi \mathrm{p}}{2}\right)\right] \\
\psi=\exp \left[\frac{\mathrm{i} \pi \mathrm{x}^{2}\left(\mathrm{a}^{4}-1\right)}{\mathrm{a}^{4} \tan \phi+\frac{1}{\tan \phi}}\right]
\end{array}
$$




\section{References}

1. G. S. Agarwal and R. Simon, "A simple realization of the fractional Fourier transform and relation to harmonic oscillator Green's function," Opt. Commun. 110 23-26 (1994).

2. L. B. Almeida, "An introduction to the angular Fourier transform," IEEE-IC ASSP Minneapolis, (1993).

3. L. B. Almeida, "The fractional Fourier transform and time-frequency representations," IEEE Transactions on Signal Processing 42 3084-3091 (1994).

4. L. M. Bernardo, O. D. D. Soares, "Fractional Fourier transforms and optical systems," Opt. Commun. 110 517-522 (1994).

5. E. U. Condon, "Immersion of the Fourier transform in a continuous group of functional transformations," Proc. Nat. Acad. Sci., U.S.A 23 158-164 (1937).

6. R. G. Dorsch, A. W. Lohmann, Y. Bitran, D. Mendlovic, and H. M. Ozaktas, "Chirp filtering in the fractional Fourier domain," Appl. Opt. 33 7599-7602 (1994).

7. D. Mendlovic, M. Ozaktas and A. W. Lohmann, "Graded index fibers, Wigner-distribution functions, and the fractional Fourier transform," Appl. Opt. 33, 6188-6193 (1994).

8. H. M. Ozaktas, B. Barshan, D. Mendlovic and L. Anural, "Convolution, filtering, and multiplexing in fractional Fourier domain and their relation to chirp and wavelet transforms," JOSA A11 547-559 (1994).

9. H. M. Ozaktas and D. Mendlovic. "Fractional Fourier transformations and their optical implementation: Part II," JOSA A10 2522-2531 (1993).

10. D. Mendlovic and H. M. Ozaktas, "Fractional Fourier transformations and their optical implementation: Part I," JOSA A10, 1875-1881 (1993).

11. H. M. Ozaktas and D. Mendlovic, "Fourier transforms of fractional orders and their optical interpretation," Opt. Commun. 101\}, 163-165 (1993).

12. E. Wigner, "On the quantum correction for thermodynamics equilibrium," Phys. Rev. 40, 749 (1932).

13. A. W. Lohmann, "Image rotation, Wigner rotation and the fractional Fourier transform," JOSA A10, 2181-2186, (1993). 